\title{
Morphological and radiometrical study of the human intervertebral foramina of the cervical spine
}

\author{
S.H. Ahmed, E.A.A. El-Shaarawy, M.F. Ishaq, M.H. Abdel Moniem \\ Department of Anatomy and Embryology, Faculty of Medicine, Cairo University, Egypt \\ [Received 24 December 2012; Accepted 17 May 2013]
}

Background: Degenerative changes of the cervical spine are an inevitable response to certain occupational status and aging processes. Compression of cervical nerve roots may result from disc degeneration, disc herniation or intervertebral foraminal stenosis. The precise and detailed anatomical knowledge of the intervertebral foramen of the cervical spine is essential for the diagnosis and management of cervical radiculopathy. The significance of the observations and findings of the present study was to elucidate the correlation between the morphology and disorders of the cervical intervertebral foramina in normal and pathological conditions especially at the level of C3-C4 to C6-C7 on both sides and in both sexes. Moreover, it will help greatly in the planning of both surgical and conservative strategies.

Materials and Methods: In the present study, 5 formalin-fixed adult cadavers and radiological specimens of the cervical region of the vertebral column of 28 normal and 209 subjects suffering from cervical disorder from both sexes and different age groups. They subjected for morphological and radiometrical analysis.

Results: All measurements of the present study of the cervical disorders in females were found to be $6 \%$ less than in males in all age groups, which is statistically significant $(p<0.01)$ as compared with the control group $(2 \%)$. The mean intervertebral foraminal areas in the control group of C5-C6 and C6-C7 are significantly greater than those of C3-C4 and C4-C5.

Conclusions: The mean intervertebral foraminal area was greater in the lower cervical region than the upper in normal adult individuals. In pathological condition the affection of C3-C4 and C4-C5 intervertebral foramina was more due to narrower surface area. The pathology of cervical spine affecting the intervertebral foramina of female which complaint earlier than male due to narrower foramina. (Folia Morphol 2014; 73, 1: 7-18)

Key words: cervical spine, cervical radiology, cervical radiometry,

\section{INTRODUCTION}

Neck pain is a common complaint all over the world. It is estimated that about $30-50 \%$ of adults will experience neck pain at a given time. It has the highest frequency in those working in hospitals and offices while those working in industrial service have the lowest frequency of neck pain [34]. The neck pain is derived from a variety of nonspecific musculoskeletal causes including progressive structural changes with or without associated systemic disease, degenerative conditions, direct trauma, chronic stress or strain injury [23]. Degenerative changes of the cervical spine are considered 
a common response to the aging process. Compression of cervical nerve roots may result from disc degeneration, disc herniation or spinal stenosis [32].

The detailed knowledge of the anatomy of the cervical intervertebral foramen (IVF) is essential for the diagnosis and management of cervical radiculopathy. Degenerative cervical disc disorder is very common. If symptoms are present, they can usually be grouped into one of 3 categories; either axial neck pain, radiculopathy, myelopathy or a combination of the three [27]. Ebraheim et al. [10] described the anatomy of the cervical neural area and divided it into 3 zones: the medial zone is the pedicle and the IVF, the middle zone is the foramen transversarium and the lateral zone is the anterior and posterior tubercles.

The anatomy of the cervical IVF has been well described in numerous literatures $[24,32,29]$. Nobuhiro et al. [24] reported that the shape of the IVF similar to a funnel; wide medially, narrow laterally and continuous with the spinal dural sheath. Lu et al. [20] compared its dimensions with normal IVF; they observed a reduction of this foramen by around $20-30 \%$ in cases suffering $1 \mathrm{~mm}$ intervertebral spaces. Moreover, these authors added that a reduction by $35-45 \%$ consequent to a $3 \mathrm{~mm}$ narrowing of intervertebral space.

Cervical spondylosis and intervertebral disc prolapse are common pathological conditions affecting some middle aged or elderly persons [2]. Moreover, cervical disc prolapse usually affects the discs between the $3^{\text {rd }}$ to the $7^{\text {th }}$ cervical intervertebral disc. Osteophytes at the intervertebral foraminal region may result in nerve compression and consequently radiculopathy $[11,31]$. Surgical approach of disorders affecting the vertebral artery, cervical spinal nerves, spinal nerve roots and the bony and ligamentous tissue related to the cervical vertebrae are safer when approached from their anterior aspect rather than posterior [29].

The present work is designed to provide a detailed study and compare the measurements and description of the anatomical and radiological features of the cervical intervertebral foramina in normal individuals of both sexes and those obtained from patients suffering from neck problem. The results obtained from this study may help in the treatment strategies of radiculopathy and cervical intervertebral disc disorders.

\section{MATERIALS AND METHODS}

Five formalin preserved adult cadavers, a total of 237 radiological cases (144 plain X-rays and 93 computed tomography [CT] scan) of the cervical region of the vertebral column of normal and pathological cases from both sexes were used and subjected for the following.

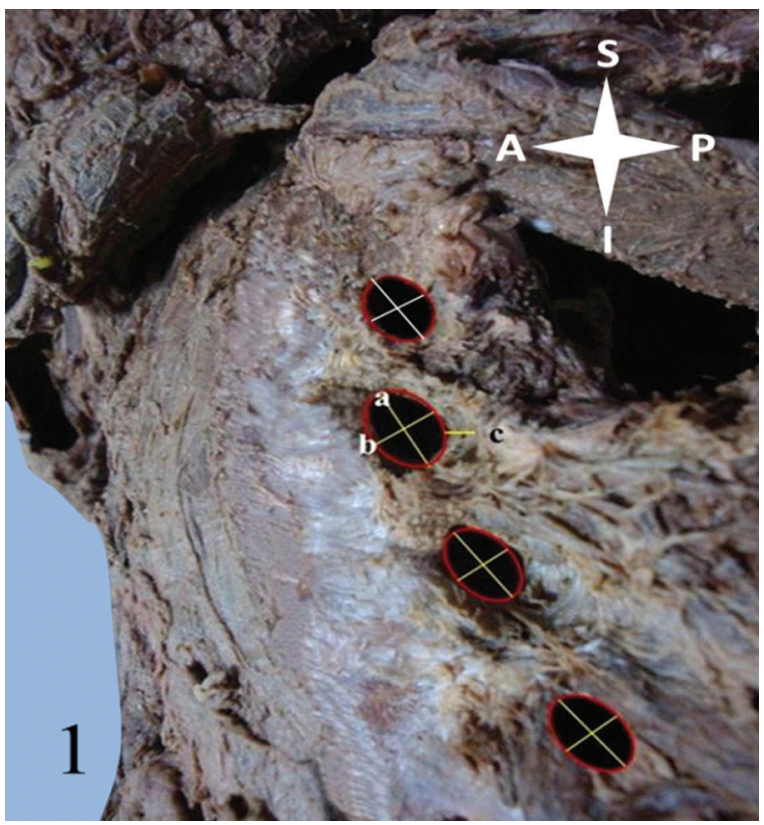

Figure 1. Dissected left side of the neck of an adult specimen showing the maximal height (a), width (b) and area (c) of the left intervertebral foramen from C3-C7.

\section{Morphological and morphometrical study}

Five formalin-fixed adult cadavers of both sexes were used. The specimens were obtained from formalinised cadavers from the Anatomy Department, Faculty of Medicine, Cairo University for gross dissection. All the specimens were apparently normal with no apparent fracture, deformities or anomalies. The specimens were subjected to:

Morphological study. The morphology of the cervical intervertebral foramina from the $3^{\text {rd }}$ to the $7^{\text {th }}$ vertebrae of both sides and the structures related to it; including the spinal nerve trunk, the blood vessels and the ligaments were carefully dissected and examined.

Morphometrical study. The studied measurements of the IVF were height and width. Assuming that the shape of the IVF is almost elliptical, according to Charles et al. [5], the maximum height of the foramen (Fig. 1A) and width (Fig. 1B) were measured using the sliding calibre (Vernier) and the area (Fig. 1C) was calculated by the following formula [5]: $\mathrm{c}=\pi \times$ $x a \times b$; where $a-$ the maximum height of the ellipse divided by $2 ; b$ - the maximum width of the ellipse divided by 2 .

\section{Radiological and radiometrical study}

The radiological cases were examined in this study (28 cases are volunteers used as normal control while 
Table 1. The number of cases and age groups examined in the current study

\begin{tabular}{|c|c|c|c|c|c|c|}
\hline \multirow[t]{2}{*}{ Group } & \multirow[t]{2}{*}{ Age [years] } & \multirow[t]{2}{*}{ No. of cases } & \multicolumn{2}{|c|}{ Normal control } & \multicolumn{2}{|c|}{ Patients with neck pain } \\
\hline & & & Male & Female & Male & Female \\
\hline I & $25-35$ & 45 & 3 & 2 & 17 & 23 \\
\hline ॥ & $36-45$ & 56 & 4 & 3 & 22 & 27 \\
\hline III & $46-55$ & 61 & 3 & 4 & 21 & 33 \\
\hline IV & $56-65$ & 75 & 5 & 4 & 31 & 35 \\
\hline
\end{tabular}

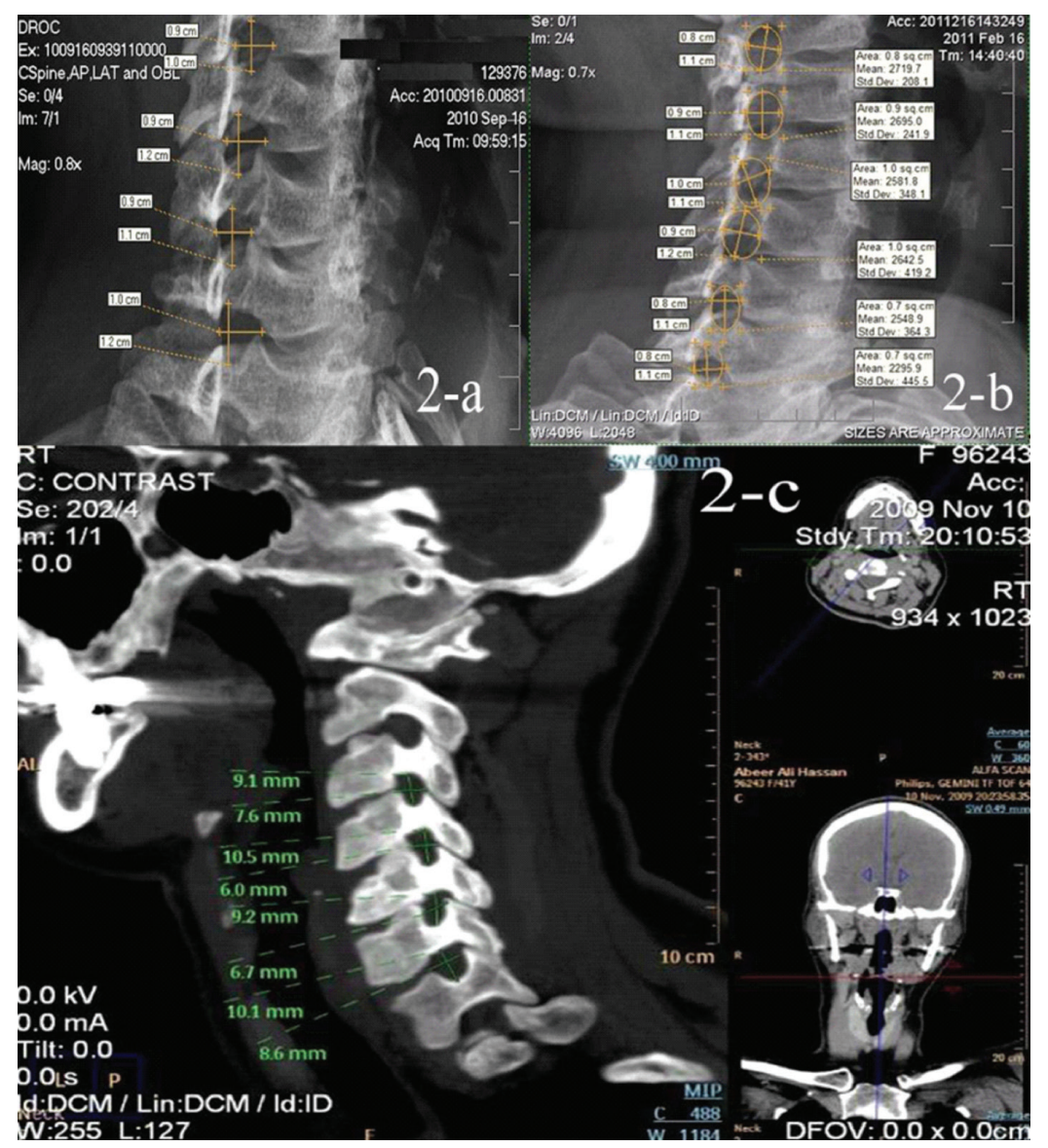

Figure 2. A. Plain X-ray (lateral view) of a male patient, 40 years old, showing the studied measurements of C3-C7 intervertebral foramina; B. Plain X-ray (lateral view) of a female patient, 35 years old, showing the studied measurements of C2-T1 intervertebral foramina; C. Computed tomography scan (oblique sagittal plane) of a female patient, 65 years old, showing the studied measurements of C3-C7 intervertebral foramina. the other 209, after taking their consent, are suffering from neck pain). The volunteers and the patients with neck pain are selected randomly and obtained from Radiology Department, Kasr El Aini Hospital; they are divided according to age into groups as in Table 1.

Plain X-rays (lateral and oblique views) and oblique-sagittal CT scans of the cervical region of different age groups of both sexes (56 males, 88 females for plain X-rays and 50 males, 43 females for CT scans) were examined for radiometrical analysis (Figs. 2A, B) using the radiological computer program (e-Film program version 2.00, 2004) and the area (Fig. 2C) was calculated by the same formula as in cadaveric study.
The CT scans of the cervical vertebrae obtained in the axial plane and reconstructed in sagittal oblique plane that is perpendicular to the direction of the intervertebral foramina in the axial plane taking in consideration the thickness of the intervertebral discs using the same radiometric program. The same measurements (Figs. 2A-C) were performed as those obtained from the plain X-ray (Fig. 2C).

\section{Statistical analysis}

Values presented as means \pm standard error of mean (SEM). Data analysed statistically by one way analysis of variance (ANOVA) followed by Scheffe's procedure pairwise comparisons and correlation 

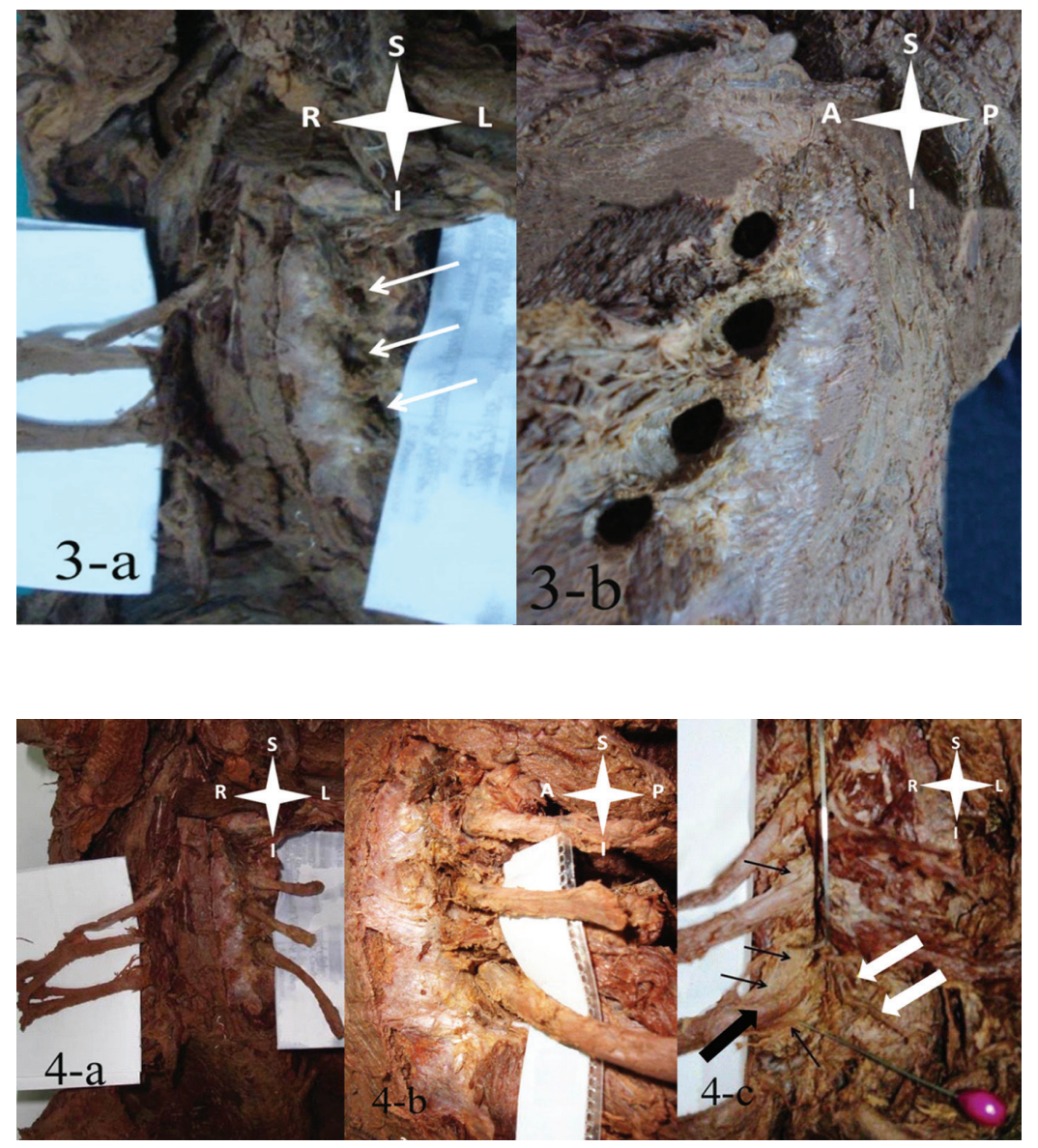

Figure 3. A. Anterior view of dissected specimen of adult neck showing almost an elliptical shaped cervical intervertebral foramen (arrows); B. Dissected right side of neck of adult specimen showing almost an elliptical shaped cervical intervertebral foramen.

Figure 4. A. Anterior view of dissected specimen of adult neck showing the cervical spinal nerves that fills most of the area of the intervertebral foramen; B. Dissected left side of neck of adult specimen, showing the cervical spinal nerves that fills most of the area of the intervertebral foramen; $\mathbf{C}$. Dissected right side of neck of adult specimen, showing the structures in the intervertebral foramen retracted and fixed by violet heads pin and silver needle. The fibrous neural sheath (thin black arrow), the spinal nerve (thick black arrow) and the blood vessels (thick white arrow).

Table 2. Mean intervertebral foraminal (IVF) height, width and area from $3^{\text {rd }}$ to $7^{\text {th }}$ cervical vertebrae of the cadaveric specimens*

\begin{tabular}{|c|c|c|c|c|c|c|}
\hline \multirow[t]{2}{*}{ Foramen level } & \multicolumn{3}{|c|}{ Left IVF } & \multicolumn{3}{|c|}{ Right IVF } \\
\hline & $\begin{array}{l}\text { Height [cm] } \\
\quad(\mathrm{n}=5)\end{array}$ & $\begin{array}{c}\text { Width [cm] } \\
\quad(\mathrm{n}=5)\end{array}$ & $\begin{array}{l}\text { Area } \\
{\left[\mathrm{cm}^{2}\right]}\end{array}$ & $\begin{array}{l}\text { Height }[\mathrm{cm}] \\
\quad(\mathrm{n}=5)\end{array}$ & $\begin{array}{c}\text { Width [cm] } \\
(\mathrm{n}=5)\end{array}$ & Area \\
\hline C3-C4 & $0.9 \pm 0.02$ & $0.8 \pm 0.04$ & $0.57 \pm 0.04$ & $0.9 \pm 0.02$ & $0.8 \pm 0.03$ & $0.57 \pm 0.04$ \\
\hline C4-C5 & $1.0 \pm 0.03$ & $0.8 \pm 0.03$ & $0.63 \pm 0.05$ & $1.1 \pm 0.03$ & $0.9 \pm 0.03$ & $0.78 \pm 0.04$ \\
\hline C5-C6 & $1.0 \pm 0.02$ & $0.8 \pm 0.03$ & $0.63 \pm 0.06$ & $1.1 \pm 0.03$ & $1.0 \pm 0.03$ & $0.86 \pm 0.05$ \\
\hline C6-C7 & $1.2 \pm 0.03$ & $0.9 \pm 0.02$ & $0.85 \pm 0.06$ & $1.3 \pm 0.04$ & $0.9 \pm 0.03$ & $0.92 \pm 0.06$ \\
\hline
\end{tabular}

${ }^{*}$ Values are presented as means $\pm \mathrm{SEM} ; \mathrm{n}$ - number of cases

using a current SPSS statistical package, and the level of significance was determined to be less than 0.05 throughout the study.

\section{RESULTS}

\section{Morphological and morphometrical study}

The current study showed that the shape of the foramen was almost elliptical in the cadaveric specimens (Fig. 3) and the spinal nerve occupying most of the area of the foramen (Fig. 4). The mean right and left intervertebral foraminal height, width and area from C3-C4 to C6-C7 were presented in Table 2. The mean intervertebral foraminal area of the foramen between C3-C4 was found to be the smallest one of both right and left sides while the foramen between C6-C7 was the largest one. The mean intervertebral foraminal areas of C4-C5 and C5-C6 were nearly similar (Table 2).

Radiological and radiometrical study. The measurements done for the radiological specimens (Fig. 5) of the normal (control) group, the mean right and left intervertebral foraminal height, width and area from C3-C4 to C6-C7 were presented in Table 3. There 


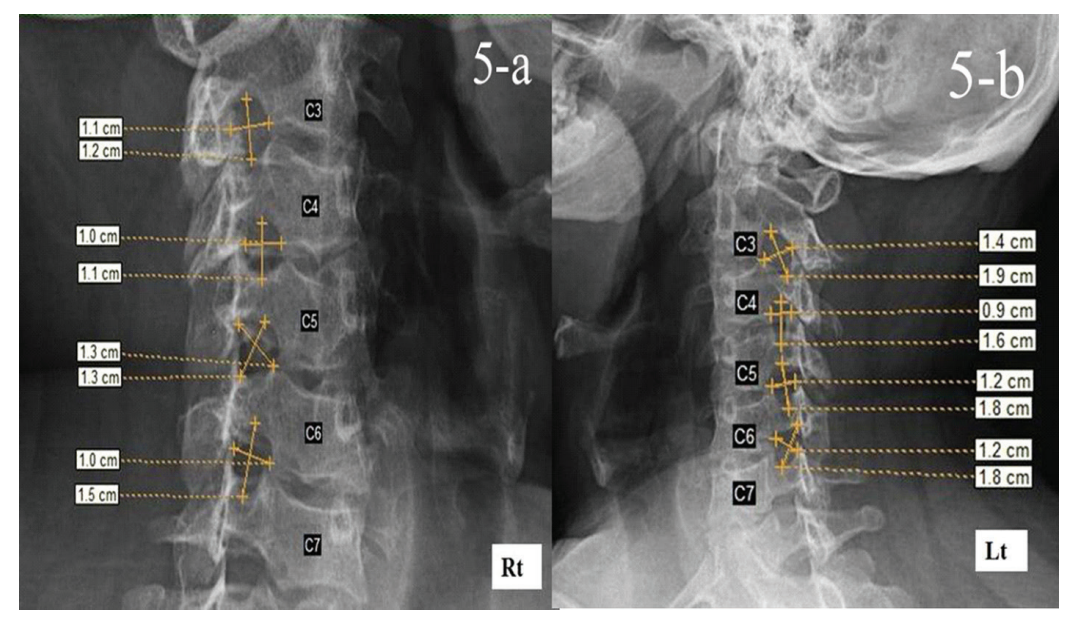

Figure 5. A. Plain X-ray (right lateral view) of a female patient, 35 years old, showing the studied measurements of C3-C7 intervertebral foramina of right side; B. Plain X-ray (left lateral view) of a female patient, 35 years old, showing the studied measurements of $\mathrm{C} 3-\mathrm{C} 7$ intervertebral foramina of the left side.

Table 3. Mean intervertebral foraminal (IVF) height, width and area from $3^{\text {rd }}$ to $7^{\text {th }}$ cervical vertebrae of the normal (control) specimens*

\begin{tabular}{lcccccccc}
\hline Foramen level & \multicolumn{3}{c}{ Left IVF } & & \multicolumn{3}{c}{ Right IVF } \\
\cline { 2 - 3 } & $\begin{array}{c}\text { Height }[\mathbf{c m}] \\
(\mathbf{n}=\mathbf{2 8})\end{array}$ & $\begin{array}{c}\text { Width [cm] } \\
(\mathbf{n}=\mathbf{2 8})\end{array}$ & & $\begin{array}{c}\text { Area } \\
{\left[\mathbf{c m}^{2}\right]}\end{array}$ & & $\begin{array}{c}\text { Height }[\mathbf{c m}] \\
(\mathbf{n}=\mathbf{2 8})\end{array}$ & $\begin{array}{c}\text { Width [cm] } \\
(\mathbf{n}=\mathbf{2 8})\end{array}$ & Area \\
\hline C3-C4 & $1.20 \pm 0.02$ & $0.91 \pm 0.02$ & & $0.78 \pm 0.04$ & & $1.12 \pm 0.02$ & $0.80 \pm 0.03$ & $0.79 \pm 0.04$ \\
C4-C5 & $1.23 \pm 0.03$ & $0.80 \pm 0.03$ & & $0.75 \pm 0.05$ & & $1.25 \pm 0.03$ & $0.75 \pm 0.03$ & $0.89 \pm 0.04$ \\
C5-C6 & $1.22 \pm 0.03$ & $090 \pm 0.04$ & & $0.82 \pm 0.06$ & & $1.19 \pm 0.03$ & $0.89 \pm 0.03$ & $0.89 \pm 0.05$ \\
C6-C7 & $1.29 \pm 0.03$ & $0.96 \pm 0.03$ & & $0.91 \pm 0.06$ & & $1.30 \pm 0.04$ & $0.95 \pm 0.03$ & $0.94 \pm 0.06$ \\
\hline
\end{tabular}

*Values are presented as means $\pm \mathrm{SEM} ; \mathrm{n}$ - number of cases

were no significant difference between the right and left sides.

The mean foraminal area between $\mathrm{C} 3-\mathrm{C} 4$ was found to be the smallest one in the right side, the mean area of the foramen between C4-C5 was found to be the smallest one in the left side while the foramen between $\mathrm{C} 6-\mathrm{C} 7$ has got the largest area in both sides (Table 3 ).

The mean right and left IVF height of the normal (control) group and pathological cases from the $3^{\text {rd }}$ to the $7^{\text {th }}$ cervical vertebrae in different age groups as regard both males and females were recorded in Tables 4 and 5 . There was no statistical significance between controls and pathological cases in age groups I, II, III. In the age group IV the mean height in control is statistically significant to that of the pathological cases.

The right mean IVF width of the normal (control) group and patients with neck pain, from $3^{\text {rd }}$ to $7^{\text {th }}$ cervical vertebrae in different age groups as regard both males and females were recorded in Tables 6 and 7. The right means width showed no statistical significance between control and pathological cases except in group III at the level of $\mathrm{C} 5-\mathrm{C} 6$ and group IV at the level of $\mathrm{C} 6-\mathrm{C} 7$. The left mean width from $\mathrm{C3}$ to $\mathrm{C7}$ in different age groups showed no statistical significance between control and pathological cases except in group IV at the level of C6-C7.

The mean intervertebral foraminal axial ratio from $3^{\text {rd }}$ to $7^{\text {th }}$ cervical vertebrae of the radiologic specimens in different age groups were calculated and recorded in Table 8. The difference between the mean axial ratio of the right and left sides is statistically significant only in the C3-C4 foramen at the age group II. The mean intervertebral foraminal areas of the normal (control) group and of the pathological cases from $3^{\text {rd }}$ to $7^{\text {th }}$ cervical vertebrae in different age groups were recorded in Tables 9 and 10.

The right and left mean areas from $\mathrm{C} 3$ to $\mathrm{C} 7$ in different age groups, in male and females and in control and pathological cases showed no statistical differences except in the last 2 levels on both sides especially in group IV (Tables 9, 10). The mean intervertebral foraminal areas in the control group of $\mathrm{C} 5-\mathrm{C} 6$ and $\mathrm{C6}-\mathrm{C} 7$ were significantly greater than that of C3-C4 and C4-C5 ( $p<0.02)$ (Table 11). It was also observed that no significant difference $(p>0.05)$ was found in the pathological group (Table 12). The mean IVF height and width in symptomatic cases were summarised in Table 13. 
Table 4. Mean intervertebral foramen height from $3^{\text {rd }}$ to $7^{\text {th }}$ cervical vertebrae of the normal (control) radiological specimens in different age groups*

\begin{tabular}{|c|c|c|c|c|c|c|c|}
\hline \multirow[t]{2}{*}{ Foramen level } & \multirow{2}{*}{$\begin{array}{l}\text { Age group } \\
\text { [years] }\end{array}$} & \multicolumn{6}{|c|}{ Mean height [cm] } \\
\hline & & $\begin{array}{l}\text { Left: female } \\
\qquad(\mathrm{n}=13)\end{array}$ & $\begin{array}{l}\text { Left: male } \\
\text { (n = 15) }\end{array}$ & $\mathbf{P}$ & $\begin{array}{l}\text { Right: female } \\
\qquad(\mathrm{n}=13)\end{array}$ & $\begin{array}{l}\text { Right: male } \\
(\mathrm{n}=15)\end{array}$ & $\mathbf{P}$ \\
\hline \multirow[t]{4}{*}{ C3-C4 } & $25-35$ & $1.13 \pm 0.03$ & $1.25 \pm 0.03$ & 0.21 & $1.14 \pm 0.05$ & $1.22 \pm 0.03$ & 0.26 \\
\hline & $36-45$ & $1.09 \pm 0.04$ & $1.28 \pm 0.03$ & 0.12 & $1.05 \pm 0.02$ & $1.20 \pm 0.04$ & 0.09 \\
\hline & $46-55$ & $1.21 \pm 0.03$ & $1.15 \pm 0.02$ & 0.44 & $1.15 \pm 0.03$ & $1.22 \pm 0.02$ & 0.14 \\
\hline & $55-65$ & $1.13 \pm 0.02$ & $0.88 \pm 0.02$ & 0.08 & $1.08 \pm 0.05$ & $0.88 \pm 0.03$ & 0.09 \\
\hline \multirow[t]{4}{*}{ C4-C5 } & $25-35$ & $1.16 \pm 0.03$ & $1.27 \pm 0.03$ & 0.17 & $1.26 \pm 0.02$ & $1.32 \pm 0.03$ & 0.29 \\
\hline & $36-45$ & $0.93 \pm 0.04$ & $1.32 \pm 0.03$ & $0.05^{* *}$ & $1.13 \pm 0.04$ & $1.29 \pm 0.04$ & 0.15 \\
\hline & $46-55$ & $1.09 \pm 0.02$ & $1.12 \pm 0.02$ & 0.27 & $1.12 \pm 0.03$ & $1.28 \pm 0.02$ & 0.12 \\
\hline & $55-65$ & $1.07 \pm 0.04$ & $1.11 \pm 0.06$ & 0.27 & $1.13 \pm 0.05$ & $1.04 \pm 0.03$ & 0.16 \\
\hline \multirow[t]{4}{*}{$\mathrm{C5}-\mathrm{C} 6$} & $25-35$ & $1.22 \pm 0.02$ & $1.16 \pm 0.02$ & 0.20 & $1.31 \pm 0.05$ & $1.26 \pm 0.04$ & 0.27 \\
\hline & $36-45$ & $1.13 \pm 0.04$ & $1.30 \pm 0.04$ & 0.10 & $1.13 \pm 0.06$ & $1.16 \pm 0.07$ & 0.33 \\
\hline & $46-55$ & $1.06 \pm 0.01$ & $1.14 \pm 0.06$ & 0.15 & $1.12 \pm 0.03$ & $1.18 \pm 0.07$ & 0.25 \\
\hline & $55-65$ & $1.23 \pm 0.06$ & $0.89 \pm 0.04$ & $0.05^{* *}$ & $1.23 \pm 0.06$ & $0.91 \pm 0.03$ & $0.04^{* *}$ \\
\hline \multirow[t]{4}{*}{$\mathrm{C6}-\mathrm{C} 7$} & $25-35$ & $1.34 \pm 0.04$ & $1.28 \pm 0.04$ & 0.29 & $1.29 \pm 0.04$ & $1.34 \pm 0.04$ & 0.19 \\
\hline & $36-45$ & $1.22 \pm 0.09$ & $1.26 \pm 0.10$ & 0.33 & $1.16 \pm 0.05$ & $1.29 \pm 0.10$ & 0.27 \\
\hline & $46-55$ & $1.11 \pm 0.07$ & $1.16 \pm 0.08$ & 0.26 & $1.19 \pm 0.04$ & $1.09 \pm 0.04$ & 0.19 \\
\hline & $55-65$ & $1.22 \pm 0.06$ & $1.08 \pm 0.05$ & 0.15 & $1.33 \pm 0.09$ & $1.11 \pm 0.04$ & $0.05^{* *}$ \\
\hline
\end{tabular}

*Values are presented as means $\pm \mathrm{SEM} ;{ }^{* *}$ Significant at $0.05 ; \mathrm{n}$ - number of cases

Table 5. Mean intervertebral foramen height from $3^{\text {rd }}$ to $7^{\text {th }}$ cervical vertebrae of the symptomatic radiological specimens in different age groups*

\begin{tabular}{|c|c|c|c|c|c|c|c|}
\hline \multirow[t]{2}{*}{ Foramen level } & \multirow{2}{*}{$\begin{array}{l}\text { Age group } \\
\text { [years] }\end{array}$} & \multicolumn{6}{|c|}{ Mean height [cm] } \\
\hline & & $\begin{array}{l}\text { Left: female } \\
\text { (n = 118) }\end{array}$ & $\begin{array}{l}\text { Left: male } \\
\text { (n = 91) }\end{array}$ & $\mathbf{P}$ & $\begin{array}{l}\text { Right: female } \\
\quad(\mathrm{n}=118)\end{array}$ & $\begin{array}{l}\text { Right: male } \\
\qquad(\mathrm{n}=91)\end{array}$ & $\mathbf{P}$ \\
\hline \multirow[t]{4}{*}{ C3-C4 } & $25-35$ & $1.10 \pm 0.02$ & $1.23 \pm 0.04$ & 0.10 & $1.10 \pm 0.02$ & $1.18 \pm 0.02$ & 0.23 \\
\hline & $36-45$ & $1.00 \pm 0.02$ & $1.25 \pm 0.03$ & $0.03^{* *}$ & $1.00 \pm 0.03$ & $1.18 \pm 0.03$ & 0.07 \\
\hline & $46-55$ & $1.08 \pm 0.02$ & $1.10 \pm 0.03$ & 0.46 & $1.12 \pm 0.02$ & $1.20 \pm 0.03$ & 0.19 \\
\hline & $55-65$ & $1.15 \pm 0.04$ & $0.90 \pm 0.03$ & $0.01 * *$ & $1.10 \pm 0.04$ & $0.90 \pm 0.04$ & $0.05^{* *}$ \\
\hline \multirow[t]{4}{*}{ C4-C5 } & $25-35$ & $1.13 \pm 0.03$ & $1.23 \pm 0.05$ & 0.06 & $1.23 \pm 0.03$ & $1.28 \pm 0.05$ & 0.34 \\
\hline & $36-45$ & $0.90 \pm 0.07$ & $1.30 \pm 0.05$ & $0.00 * *$ & $1.10 \pm 0.07$ & $1.25 \pm 0.05$ & 0.10 \\
\hline & $46-55$ & $1.08 \pm 0.02$ & $1.10 \pm 0.04$ & 0.38 & $1.10 \pm 0.02$ & $1.25 \pm 0.04$ & 0.06 \\
\hline & $55-65$ & $1.08 \pm 0.09$ & $1.10 \pm 0.05$ & 0.45 & $1.15 \pm 0.09$ & $1.02 \pm 0.05$ & $0.02^{* *}$ \\
\hline \multirow[t]{4}{*}{$\mathrm{C5}-\mathrm{C6}$} & $25-35$ & $1.20 \pm 0.05$ & $1.10 \pm 0.04$ & 0.20 & $1.28 \pm 0.05$ & $1.23 \pm 0.04$ & 0.30 \\
\hline & $36-45$ & $1.10 \pm 0.06$ & $1.28 \pm 0.07$ & 0.10 & $1.10 \pm 0.06$ & $1.15 \pm 0.07$ & 0.38 \\
\hline & $46-55$ & $1.02 \pm 0.03$ & $1.10 \pm 0.07$ & 0.15 & $1.10 \pm 0.03$ & $1.15 \pm 0.07$ & 0.39 \\
\hline & $55-65$ & $1.25 \pm 0.09$ & $0.90 \pm 0.09$ & $0.03 * *$ & $1.25 \pm 0.06$ & $0.93 \pm 0.03$ & $0.01 * *$ \\
\hline \multirow[t]{4}{*}{$\mathrm{C6}-\mathrm{C7}$} & $25-35$ & $1.30 \pm 0.04$ & $1.25 \pm 0.04$ & 0.27 & $1.23 \pm 0.04$ & $1.28 \pm 0.04$ & 0.28 \\
\hline & $36-45$ & $1.20 \pm 0.09$ & $1.22 \pm 0.10$ & 0.47 & $1.10 \pm 0.05$ & $1.25 \pm 0.10$ & 0.26 \\
\hline & $46-55$ & $1.10 \pm 0.07$ & $1.15 \pm 0.08$ & 0.50 & $1.18 \pm 0.04$ & $1.00 \pm 0.04$ & 0.17 \\
\hline & $55-65$ & $1.25 \pm 0.06$ & $1.10 \pm 0.05$ & 0.10 & $1.35 \pm 0.09$ & $1.10 \pm 0.04$ & $0.00^{* *}$ \\
\hline
\end{tabular}

*Values are presented as means $\pm \mathrm{SEM}$; * Significant at $0.05 ; \mathrm{n}$ - number of cases 
Table 6. Mean intervertebral foramen width from $3^{\text {rd }}$ to $7^{\text {th }}$ cervical vertebrae of the normal (control) radiological specimens in different age groups*

\begin{tabular}{|c|c|c|c|c|c|c|c|}
\hline \multirow[t]{2}{*}{ Foramen level } & \multirow{2}{*}{$\begin{array}{l}\text { Age group } \\
\text { [years] }\end{array}$} & \multicolumn{6}{|c|}{ Mean width [cm] } \\
\hline & & $\begin{array}{l}\text { Left: female } \\
\qquad(\mathrm{n}=13)\end{array}$ & $\begin{array}{l}\text { Left: male } \\
\text { (n = 15) }\end{array}$ & $\mathbf{P}$ & $\begin{array}{l}\text { Right: female } \\
\quad(\mathrm{n}=13)\end{array}$ & $\begin{array}{l}\text { Right: male } \\
\quad(\mathrm{n}=15)\end{array}$ & $\mathbf{P}$ \\
\hline \multirow[t]{4}{*}{ C3-C4 } & $25-35$ & $0.94 \pm 0.03$ & $0.89 \pm 0.03$ & 0.24 & $0.91 \pm 0.04$ & $0.99 \pm 0.02$ & 0.27 \\
\hline & $36-45$ & $0.93 \pm 0.05$ & $0.82 \pm 0.02$ & 0.19 & $0.93 \pm 0.03$ & $0.86 \pm 0.05$ & 0.22 \\
\hline & $46-55$ & $0.83 \pm 0.03$ & $0.85 \pm 0.04$ & 0.22 & $0.77 \pm 0.02$ & $0.98 \pm 0.03$ & 0.10 \\
\hline & $55-65$ & $0.83 \pm 0.02$ & $0.72 \pm 0.02$ & 0.14 & $0.86 \pm 0.05$ & $0.76 \pm 0.02$ & 0.26 \\
\hline \multirow[t]{4}{*}{ C4-C5 } & $25-35$ & $0.88 \pm 0.02$ & $0.94 \pm 0.02$ & 0.22 & $0.96 \pm 0.03$ & $1.06 \pm 0.05$ & 0.22 \\
\hline & $36-45$ & $0.92 \pm 0.05$ & $0.86 \pm 0.04$ & 0.25 & $0.94 \pm 0.07$ & $0.97 \pm 0.05$ & 0.31 \\
\hline & $46-55$ & $0.81 \pm 0.03$ & $0.79 \pm 0.02$ & 0.33 & $0.86 \pm 0.02$ & $0.97 \pm 0.04$ & 0.12 \\
\hline & $55-65$ & $0.88 \pm 0.06$ & $0.80 \pm 0.03$ & 0.21 & $0.86 \pm 0.09$ & $0.72 \pm 0.05$ & 0.24 \\
\hline \multirow[t]{4}{*}{ C5-C6 } & $25-35$ & $1.09 \pm 0.03$ & $0.90 \pm 0.02$ & 0.11 & $0.98 \pm 0.01$ & $0.89 \pm 0.03$ & 0.10 \\
\hline & $36-45$ & $0.93 \pm 0.02$ & $0.89 \pm 0.04$ & 0.23 & $0.84 \pm 0.02$ & $0.90 \pm 0.05$ & 0.23 \\
\hline & $46-55$ & $0.68 \pm 0.05$ & $0.79 \pm 0.05$ & 0.22 & $0.75 \pm 0.03$ & $0.97 \pm 0.03$ & $0.05^{* *}$ \\
\hline & $55-65$ & $0.96 \pm 0.06$ & $0.82 \pm 0.06$ & 0.12 & $1.02 \pm 0.04$ & $0.66 \pm 0.03$ & 0.22 \\
\hline \multirow[t]{4}{*}{ C6-C7 } & $25-35$ & $1.09 \pm 0.03$ & $0.99 \pm 0.03$ & 0.12 & $1.07 \pm 0.02$ & $0.99 \pm 0.03$ & 0.22 \\
\hline & $36-45$ & $0.76 \pm 0.04$ & $0.90 \pm 0.03$ & 0.22 & $1.05 \pm 0.09$ & $0.98 \pm 0.12$ & 0.12 \\
\hline & $46-55$ & $0.83 \pm 0.04$ & $0.84 \pm 0.04$ & 0.25 & $0.89 \pm 0.07$ & $0.92 \pm 0.08$ & 0.25 \\
\hline & $55-65$ & $0.98 \pm 0.06$ & $0.69 \pm 0.02$ & $0.04^{* *}$ & $1.02 \pm 0.06$ & $0.81 \pm 0.05$ & $0.05^{* *}$ \\
\hline
\end{tabular}

*Values are presented as means $\pm \mathrm{SEM} ;{ }^{* *}$ Significant at $0.05 ; n$ - number of cases

Table 7. Mean intervertebral foramen width from $3^{\text {rd }}$ to $7^{\text {th }}$ cervical vertebrae of the symptomatic radiological specimens in different age groups*

\begin{tabular}{|c|c|c|c|c|c|c|c|}
\hline \multirow[t]{2}{*}{ Foramen level } & \multirow{2}{*}{$\begin{array}{l}\text { Age group } \\
\text { [years] }\end{array}$} & \multicolumn{6}{|c|}{ Mean width [cm] } \\
\hline & & $\begin{array}{l}\text { Left: female } \\
\text { (n = 118) }\end{array}$ & $\begin{array}{l}\text { Left: male } \\
\text { (n = 91) }\end{array}$ & $\mathbf{P}$ & $\begin{array}{l}\text { Right: female } \\
\qquad(\mathrm{n}=118)\end{array}$ & $\begin{array}{l}\text { Right: male } \\
\quad(\mathrm{n}=91)\end{array}$ & $\mathbf{P}$ \\
\hline \multirow[t]{4}{*}{ C3-C4 } & $25-35$ & $0.90 \pm 0.02$ & $0.85 \pm 0.04$ & 0.27 & $0.88 \pm 0.02$ & $0.95 \pm 0.04$ & 0.27 \\
\hline & $36-45$ & $0.90 \pm 0.02$ & $0.77 \pm 0.03$ & 0.21 & $0.90 \pm 0.02$ & $0.82 \pm 0.03$ & 0.22 \\
\hline & $46-55$ & $0.80 \pm 0.02$ & $0.80 \pm 0.03$ & 0.50 & $0.75 \pm 0.02$ & $0.95 \pm 0.03$ & 0.10 \\
\hline & $55-65$ & $0.85 \pm 0.04$ & $0.74 . \pm 0.03$ & 0.23 & $0.85 \pm 0.04$ & $0.75 . \pm 0.03$ & 0.26 \\
\hline \multirow[t]{4}{*}{ C4-C5 } & $25-35$ & $0.85 \pm 0.03$ & $0.90 \pm 0.05$ & 0.31 & $0.93 \pm 0.03$ & $0.98 \pm 0.05$ & 0.34 \\
\hline & $36-45$ & $0.90 \pm 0.07$ & $0.82 \pm 0.05$ & 0.25 & $0.90 \pm 0.07$ & $0.93 \pm 0.05$ & 0.43 \\
\hline & $46-55$ & $0.78 \pm 0.02$ & $0.75 \pm 0.04$ & 0.42 & $0.85 \pm 0.02$ & $0.95 \pm 0.04$ & 0.21 \\
\hline & $55-65$ & $0.90 \pm 0.09$ & $0.80 \pm 0.05$ & 0.15 & $0.88 \pm 0.09$ & $0.70 \pm 0.05$ & 0.06 \\
\hline \multirow[t]{4}{*}{ C5-C6 } & $25-35$ & $1.05 \pm 0.05$ & $0.83 \pm 0.04$ & $0.00^{* *}$ & $0.95 \pm 0.05$ & $0.85 \pm 0.04$ & 0.10 \\
\hline & $36-45$ & $0.90 \pm 0.06$ & $0.85 \pm 0.07$ & 0.40 & $0.80 \pm 0.06$ & $0.87 \pm 0.07$ & 0.32 \\
\hline & $46-55$ & $0.67 \pm 0.03$ & $0.75 \pm 0.07$ & 0.26 & $0.73 \pm 0.03$ & $0.95 \pm 0.07$ & $0.05^{* *}$ \\
\hline & $55-65$ & $1.00 \pm 0.09$ & $0.84 \pm 0.09$ & $0.03^{* *}$ & $1.08 \pm 0.09$ & $0.69 \pm 0.09$ & $0.01^{* *}$ \\
\hline \multirow[t]{4}{*}{$\mathrm{C6}-\mathrm{C7}$} & $25-35$ & $1.05 \pm 0.04$ & $0.93 \pm 0.04$ & 0.09 & $1.00 \pm 0.04$ & $0.95 \pm 0.04$ & 0.29 \\
\hline & $36-45$ & $0.70 \pm 0.09$ & $0.87 \pm 0.10$ & 0.17 & $1.00 \pm 0.09$ & $0.95 \pm 0.10$ & 0.38 \\
\hline & $46-55$ & $0.80 \pm 0.07$ & $0.80 \pm 0.08$ & 0.50 & $0.87 \pm 0.07$ & $0.90 \pm 0.08$ & 0.44 \\
\hline & $55-65$ & $1.03 \pm 0.06$ & $0.70 \pm 0.05$ & $0.00^{* *}$ & $1.05 \pm 0.06$ & $0.80 \pm 0.05$ & $0.03^{* *}$ \\
\hline
\end{tabular}

\footnotetext{
*Values are presented as means $\pm \mathrm{SEM} ;{ }^{*}$ Significant at $0.05 ; \mathrm{n}$ - number of cases
} 
Table 8. Mean intervertebral foraminal axial ratio from $3^{\text {rd }}$ to $7^{\text {th }}$ cervical vertebrae of the symptomatic radiological specimens in different age groups*

\begin{tabular}{|c|c|c|c|c|}
\hline \multirow[t]{2}{*}{ Foramen level } & \multirow{2}{*}{$\begin{array}{l}\text { Age group } \\
\text { [years] }\end{array}$} & \multicolumn{3}{|c|}{ Axial ratio } \\
\hline & & Left $(n=209)$ & Right (n= 209) & $\mathbf{P}$ \\
\hline \multirow[t]{4}{*}{ C3-C4 } & $25-35$ & $1.3 \pm 0.06$ & $1.4 \pm 0.05$ & 0.194 \\
\hline & $36-45$ & $1.8 \pm 0.07$ & $1.4 \pm 0.05$ & $0.026^{* *}$ \\
\hline & $46-55$ & $1.5 \pm 0.04$ & $1.5 \pm 0.05$ & 0.446 \\
\hline & $55-65$ & $1.5 \pm 0.05$ & $1.6 \pm 0.06$ & 0.100 \\
\hline \multirow[t]{4}{*}{ C4-C5 } & $25-35$ & $1.4 \pm 0.04$ & $1.2 \pm 0.04$ & 0.118 \\
\hline & $36-45$ & $1.5 \pm 0.04$ & $1.5 \pm 0.05$ & 0.127 \\
\hline & $46-55$ & $1.4 \pm 0.04$ & $1.3 \pm 0.04$ & 0.154 \\
\hline & $55-65$ & $1.3 \pm 0.04$ & $1.4 \pm 0.05$ & 0.177 \\
\hline \multirow[t]{4}{*}{$\mathrm{C5}-\mathrm{C6}$} & $25-35$ & $1.2 \pm 0.03$ & $1.3 \pm 0.04$ & 0.102 \\
\hline & $36-45$ & $1.3 \pm 0.03$ & $1.4 \pm 0.04$ & 0.378 \\
\hline & $46-55$ & $1.5 \pm 0.04$ & $1.4 \pm 0.04$ & 0.240 \\
\hline & $55-65$ & $1.2 \pm 0.03$ & $1.3 \pm 0.02$ & 0.476 \\
\hline \multirow[t]{4}{*}{$\mathrm{C} 6-\mathrm{C} 7$} & $25-35$ & $1.2 \pm 0.03$ & $1.2 \pm 0.03$ & 0.371 \\
\hline & $36-45$ & $1.4 \pm 0.04$ & $1.3 \pm 0.04$ & 0.192 \\
\hline & $46-55$ & $1.4 \pm 0.04$ & $1.3 \pm 0.05$ & 0.178 \\
\hline & $55-65$ & $1.3 \pm 0.03$ & $1.1 \pm 0.02$ & 0.081 \\
\hline
\end{tabular}

*Values are presented as means $\pm \mathrm{SEM} ;{ }^{* *}$ Significant at $0.05 ; \mathrm{n}$ - number of cases

Table 9. Mean intervertebral foraminal area from $3^{\text {rd }}$ to $7^{\text {th }}$ cervical vertebrae of the normal (control) radiological specimens in different age groups*

\begin{tabular}{|c|c|c|c|c|c|c|c|}
\hline \multirow[t]{2}{*}{ Foramen level } & \multirow{2}{*}{$\begin{array}{l}\text { Age group } \\
\text { [years] }\end{array}$} & \multicolumn{6}{|c|}{ Mean area } \\
\hline & & $\begin{array}{c}\text { Left: } \\
\text { male } \\
\text { (n=15) }\end{array}$ & $\begin{array}{c}\text { Left: } \\
\text { female } \\
\text { (n=13) }\end{array}$ & $\mathbf{P}$ & $\begin{array}{c}\text { Right: } \\
\text { male } \\
\text { (n=15) }\end{array}$ & $\begin{array}{c}\text { Right: } \\
\text { female } \\
\text { (n=13) }\end{array}$ & $\mathbf{P}$ \\
\hline \multirow[t]{4}{*}{ C3-C4 } & $25-35$ & $0.86 \pm 0.03$ & $0.80 \pm 0.05$ & 0.34 & $0.92 \pm 0.05$ & $0.78 \pm 0.04$ & 0.20 \\
\hline & $36-45$ & $0.81 \pm 0.02$ & $0.74 \pm 0.03$ & 0.36 & $0.79 \pm 0.04$ & $0.74 \pm 0.04$ & 0.26 \\
\hline & $46-55$ & $0.72 \pm 0.02$ & $0.70 \pm 0.01$ & 0.45 & $0.93 \pm 0.05$ & $0.69 \pm 0.02$ & $0.04^{* *}$ \\
\hline & $55-65$ & $0.63 \pm 0.04$ & $0.75 \pm 0.04$ & 0.06 & $0.63 \pm 0.03$ & $0.72 \pm 0.04$ & 0.11 \\
\hline \multirow[t]{4}{*}{ C4-C5 } & $25-35$ & $0.91 \pm 0.03$ & $0.80 \pm 0.02$ & 0.19 & $0.99 \pm 0.04$ & $0.92 \pm 0.04$ & 0.28 \\
\hline & $36-45$ & $0.89 \pm 0.04$ & $0.71 \pm 0.04$ & 0.22 & $0.96 \pm 0.03$ & $0.82 \pm 0.02$ & 0.31 \\
\hline & $46-55$ & $0.69 \pm 0.03$ & $0.75 \pm 0.03$ & 0.35 & $0.95 \pm 0.04$ & $0.73 \pm 0.03$ & 0.09 \\
\hline & $55-65$ & $0.67 \pm 0.02$ & $0.76 \pm 0.03$ & 0.28 & $0.62 \pm 0.02$ & $0.75 \pm 0.05$ & 0.07 \\
\hline \multirow[t]{4}{*}{$\mathrm{C5}-\mathrm{C} 6$} & $25-35$ & $0.77 \pm 0.04$ & $0.98 \pm 0.05$ & $0.01 * *$ & $0.96 \pm 0.04$ & $1.05 \pm 0.05$ & 0.16 \\
\hline & $36-45$ & $0.91 \pm 0.02$ & $0.82 \pm 0.04$ & 0.23 & $0.86 \pm 0.04$ & $0.72 \pm 0.04$ & 0.29 \\
\hline & $46-55$ & $0.69 \pm 0.03$ & $0.65 \pm 0.02$ & 0.32 & $0.88 \pm 0.04$ & $0.69 \pm 0.03$ & 0.21 \\
\hline & $55-65$ & $0.80 \pm 0.02$ & $0.95 \pm 0.03$ & $0.02^{* *}$ & $0.85 \pm 0.02$ & $0.96 \pm 0.04$ & $0.00^{* *}$ \\
\hline \multirow[t]{4}{*}{$\mathrm{C} 6-\mathrm{C} 7$} & $25-35$ & $0.95 \pm 0.03$ & $1.09 \pm 0.02$ & 0.16 & $0.99 \pm 0.03$ & $1.02 \pm 0.04$ & 0.39 \\
\hline & $36-45$ & $0.88 \pm 0.04$ & $0.69 \pm 0.04$ & 0.27 & $0.99 \pm 0.04$ & $0.90 \pm 0.02$ & 0.35 \\
\hline & $46-55$ & $0.75 \pm 0.05$ & $0.73 \pm 0.02$ & 0.39 & $0.75 \pm 0.05$ & $0.86 \pm 0.02$ & 0.23 \\
\hline & $55-65$ & $0.62 \pm 0.04$ & $0.98 \pm 0.02$ & $0.00^{* *}$ & $0.67 \pm 0.01$ & $1.08 \pm 0.04$ & $0.01^{* *}$ \\
\hline
\end{tabular}

*Values are presented as means $\pm \mathrm{SEM} ;{ }^{* *}$ Significant at $0.05 ; \mathrm{n}$ - number of cases 
Table 10. Mean intervertebral foraminal area from $3^{\text {rd }}$ to $7^{\text {th }}$ cervical vertebrae of the symptomatic radiological specimens in different age groups*

\begin{tabular}{|c|c|c|c|c|c|c|c|}
\hline \multirow[t]{2}{*}{ Foramen level } & \multirow{2}{*}{$\begin{array}{l}\text { Age group } \\
\text { [years] }\end{array}$} & \multicolumn{6}{|c|}{ Mean area } \\
\hline & & $\begin{array}{c}\text { Left: } \\
\text { male } \\
\text { (n=91) }\end{array}$ & $\begin{array}{c}\text { Left: } \\
\text { female } \\
\text { (n=118) }\end{array}$ & $\mathbf{P}$ & $\begin{array}{c}\text { Right: } \\
\text { male } \\
\text { (n= 91) }\end{array}$ & $\begin{array}{c}\text { Right: } \\
\text { female } \\
\text { (n= 118) }\end{array}$ & $\mathbf{P}$ \\
\hline \multirow[t]{4}{*}{ C3-C4 } & $25-35$ & $0.82 \pm 0.04$ & $0.78 \pm 0.03$ & 0.32 & $0.88 \pm 0.05$ & $0.75 \pm 0.04$ & 0.18 \\
\hline & $36-45$ & $0.77 \pm 0.03$ & $0.71 \pm 0.03$ & 0.39 & $0.76 \pm 0.04$ & $0.71 \pm 0.04$ & 0.34 \\
\hline & $46-55$ & $0.69 \pm 0.02$ & $0.70 \pm 0.02$ & 0.49 & $0.91 \pm 0.05$ & $0.66 \pm 0.02$ & $0.05^{* *}$ \\
\hline & $55-65$ & $0.62 \pm 0.02$ & $0.77 \pm 0.04$ & 0.08 & $0.64 \pm 0.03$ & $0.73 \pm 0.04$ & 0.11 \\
\hline \multirow[t]{4}{*}{ C4-C5 } & $25-35$ & $0.87 \pm 0.04$ & $0.75 \pm 0.04$ & 0.11 & $0.87 \pm 0.06$ & $0.79 \pm 0.05$ & 0.30 \\
\hline & $36-45$ & $0.85 \pm 0.04$ & $0.64 \pm 0.02$ & 0.18 & $0.83 \pm 0.05$ & $0.78 \pm 0.03$ & 0.26 \\
\hline & $46-55$ & $0.65 \pm 0.04$ & $0.74 \pm 0.03$ & 0.44 & $0.84 \pm 0.05$ & $0.67 \pm 0.02$ & 0.11 \\
\hline & $55-65$ & $0.69 \pm 0.02$ & $0.80 \pm 0.04$ & 0.35 & $0.70 \pm 0.04$ & $0.77 \pm 0.04$ & 0.11 \\
\hline \multirow[t]{4}{*}{$\mathrm{C5}-\mathrm{C6}$} & $25-35$ & $0.72 \pm 0.03$ & $0.96 \pm 0.04$ & $0.03^{* *}$ & $0.82 \pm 0.04$ & $0.99 \pm 0.05$ & 0.12 \\
\hline & $36-45$ & $0.88 \pm 0.04$ & $0.78 \pm 0.03$ & 0.36 & $0.81 \pm 0.04$ & $0.69 \pm 0.03$ & 0.31 \\
\hline & $46-55$ & $0.66 \pm 0.02$ & $0.63 \pm 0.02$ & 0.17 & $0.85 \pm 0.04$ & $0.65 \pm 0.02$ & 0.17 \\
\hline & $55-65$ & $0.64 \pm 0.02$ & $0.98 \pm 0.05$ & $0.00^{* *}$ & $0.80 \pm 0.02$ & $1.06 \pm 0.06$ & $0.01 * *$ \\
\hline \multirow[t]{4}{*}{$\mathrm{C} 6-\mathrm{C} 7$} & $25-35$ & $0.86 \pm 0.04$ & $1.07 \pm 0.05$ & 0.11 & $0.95 \pm 0.05$ & $0.97 \pm 0.05$ & 0.44 \\
\hline & $36-45$ & $0.85 \pm 0.03$ & $0.66 \pm 0.02$ & 0.25 & $0.96 \pm 0.05$ & $0.86 \pm 0.04$ & 0.38 \\
\hline & $46-55$ & $0.71 \pm 0.02$ & $0.71 \pm 0.03$ & 0.49 & $0.71 \pm 0.03$ & $0.84 \pm 0.04$ & 0.34 \\
\hline & $55-65$ & $0.60 \pm 0.02$ & $1.01 \pm 0.05$ & $0.01 * *$ & $0.69 \pm 0.02$ & $1.12 \pm 0.06$ & $0.02^{* *}$ \\
\hline
\end{tabular}

*Values are presented as means $\pm \mathrm{SEM}$; * Significant at $0.05 ; \mathrm{n}$ - number of cases

Table 11. Mean intervertebral foraminal (IVF) area from $3^{\text {rd }}$ to $7^{\text {th }}$ cervical vertebrae of the normal (control) specimens*

\begin{tabular}{lccc}
\hline Foramen & Right IVF & Left IVF & P \\
\hline C3-C4 & $0.82 \pm 0.02$ & $0.75 \pm 0.04$ & $0.03^{* *}$ \\
C4-C5 & $0.88 \pm 0.04$ & $0.79 \pm 0.02$ & $0.02^{* *}$ \\
\hline C5-C6 & $0.90 \pm 0.02$ & $0.79 \pm 0.03$ & $0.01^{* *}$ \\
C6-C7 & $0.96 \pm 0.03$ & $0.80 \pm 0.04$ & $0.00^{* *}$ \\
\hline
\end{tabular}

*Values are presented as means \pm SEM; **Significant at 0.05

\section{DISCUSSION}

The present study demonstrated the morphological and radiometrical features of the cervical intervertebral foramina from $\mathrm{C} 3-\mathrm{C} 4$ to $\mathrm{C} 6-\mathrm{C} 7$ on both sides and sexes. The dimensions of the foramen are of clinical importance in the diagnosis of foraminal stenosis and radiculopathy.

The purpose of the study is to describe the dimensions of the cervical IVF in the neutral position in a sample of asymptomatic adults (28 cases) and a wide range of radiological specimens of patients complaining of radicular symptoms (209 cases). The cervical intervertebral foramina have been previously described by Ruggieri [30] as becoming progressively narrower in the lower segments normally. The fin-
Table 12. Mean intervertebral foraminal (IVF) area from $3^{\text {rd }}$ to $7^{\text {th }}$ cervical vertebrae of the pathological specimens*

\begin{tabular}{lccc}
\hline Foramen & Right IVF & Left IVF & P \\
\hline C3-C4 & $0.80 \pm 0.02$ & $0.73 \pm 0.04$ & $0.03^{* *}$ \\
C4-C5 & $0.81 \pm 0.04$ & $0.77 \pm 0.02$ & $0.05^{* *}$ \\
\hline C5-C6 & $0.82 \pm 0.02$ & $0.73 \pm 0.03$ & $0.01^{* *}$ \\
C6-C7 & $0.83 \pm 0.03$ & $0.75 \pm 0.04$ & $0.01^{* *}$ \\
\hline
\end{tabular}

*Values are presented as means \pm SEM; ${ }^{* *}$ Significant at 0.05

dings of the present study generally do not support this conclusion as there is no progressive decrease in the foraminal dimensions normally with each progressive caudal segment.

Czervionke et al. [8] reported that the cervical intervertebral foramina are oval in shape; the width of the foramen is approximately one half its heights. This study supports the conclusion that the foramen is elliptical in shape. However, it is found that the width of the foramen is approximately two thirds its heights across all levels. The results for the height and width of the foramen in this study are based upon a single measurement defined simply as the greatest vertical and horizontal diameters at right angle to each other. These variables do not specifically address changes 
Table 13. Mean intervertebral foramen height and width from $3^{\text {rd }}$ to $7^{\text {th }}$ cervical vertebrae of the symptomatic radiological specimens in different age groups*

\begin{tabular}{|c|c|c|c|c|c|}
\hline \multirow[t]{2}{*}{ Foramen level } & \multirow{2}{*}{$\begin{array}{l}\text { Age group } \\
\text { [years] }\end{array}$} & \multicolumn{2}{|c|}{ Mean height [cm] } & \multicolumn{2}{|c|}{ Mean width [cm] } \\
\hline & & Left $(n=209)$ & Right (n = 209) & Left $(n=209)$ & Right $(n=209)$ \\
\hline \multirow[t]{4}{*}{ C3-C4 } & $25-35$ & $1.14 \pm 0.02$ & $1.13 \pm 0.02$ & $0.82 \pm 0.03$ & $0.84 \pm 0.03$ \\
\hline & $36-45$ & $1.15 \pm 0.03$ & $1.19 \pm 0.03$ & $0.84 \pm 0.03$ & $0.90 \pm 0.03$ \\
\hline & $46-55$ & $1.15 \pm 0.02$ & $1.17 \pm 0.03$ & $0.85 \pm 0.04$ & $0.87 \pm 0.03$ \\
\hline & $55-65$ & $1.20 \pm 0.04$ & $1.22 \pm 0.04$ & $0.89 \pm 0.03$ & $0.95 \pm 0.03$ \\
\hline \multirow[t]{4}{*}{ C4-C5 } & $25-35$ & $1.17 \pm 0.03$ & $1.25 \pm 0.05$ & $0.88 \pm 0.05$ & $0.95 \pm 0.05$ \\
\hline & $36-45$ & $1.24 \pm 0.07$ & $1.23 \pm 0.05$ & $0.83 \pm 0.07$ & $0.93 \pm 0.08$ \\
\hline & $46-55$ & $1.09 \pm 0.02$ & $1.14 \pm 0.04$ & $0.78 \pm 0.07$ & $0.88 \pm 0.05$ \\
\hline & $55-65$ & $1.08 \pm 0.09$ & $1.14 \pm 0.05$ & $0.88 \pm 0.05$ & $0.84 \pm 0.06$ \\
\hline \multirow[t]{4}{*}{ C5-C6 } & $25-35$ & $1.15 \pm 0.05$ & $1.25 \pm 0.04$ & $0.94 \pm 0.05$ & $0.90 \pm 0.03$ \\
\hline & $36-45$ & $1.25 \pm 0.06$ & $1.14 \pm 0.07$ & $0.86 \pm 0.09$ & $0.86 \pm 0.06$ \\
\hline & $46-55$ & $1.04 \pm 0.03$ & $1.11 \pm 0.07$ & $0.69 \pm 0.05$ & $0.79 \pm 0.06$ \\
\hline & $55-65$ & $1.18 \pm 0.09$ & $1.18 \pm 0.09$ & $0.96 \pm 0.05$ & $0.98 \pm 0.10$ \\
\hline \multirow[t]{4}{*}{$\mathrm{C} 6-\mathrm{C} 7$} & $25-35$ & $1.28 \pm 0.04$ & $1.25 \pm 0.04$ & $0.99 \pm 0.04$ & $0.98 \pm 0.03$ \\
\hline & $36-45$ & $1.21 \pm 0.09$ & $1.23 \pm 0.10$ & $0.84 \pm 0.08$ & $0.96 \pm 0.03$ \\
\hline & $46-55$ & $1.10 \pm 0.07$ & $1.14 \pm 0.08$ & $0.80 \pm 0.06$ & $0.88 \pm 0.03$ \\
\hline & $55-65$ & $1.22 \pm 0.06$ & $1.30 \pm 0.05$ & $0.96 \pm 0.07$ & $1.02 \pm 0.07$ \\
\hline
\end{tabular}

${ }^{*}$ Values are presented as means $\pm \mathrm{SEM} ; \mathrm{n}$ - number of cases

in width for the lower third of the foraminal space where the nerve root resides and concerns of stenosis are greatest [15]. For the separate variables of foraminal height, width and area paired t-test was used to determine the significant differences, in foraminal dimensions between right and left sides and across sexes in the cervical neutral position. In the present study all measurements of the pathological cases in females were found to be $6 \%$ less than in males in all age groups, which is statistically significant $(p<0.01)$ compared with the control one $(2 \%)$. The same findings were reported by Hogg-Johnson et al. [12] who found that the intervertebral foraminal areas in women were only slightly smaller than in men.

The anatomical structures within the IVF are well described in previous studies $[7,18,25]$. These structures consist primarily of the anterior and posterior roots, spinal ganglion, spinal arteries and veins, the ligamenta flava and loose connective fatty tissue. The connective tissues in the IVF occupy less than $28 \%$ and the neural tissues apparently less than $35 \%$ of the IVF's overall content while in the current study it is found that the neural content occupies more than $60 \%$ of the foraminal area. However, Moeti and Marchetti [21] mentioned that the dorsal root ganglion is just slightly smaller than the whole IVF. The last authors described the dorsal root ganglion as lying in the superolateral area of the foramen and often play a significant role as a sensation trigger in neck pain.

The anatomy of the cervical IVF has been well described in several studies $[10,18,24,25]$. Ebraheim et al. [10] described the quantitative anatomy of the cervical nerve root groove and divided it into 3 zones: the medial zone (pedicle), middle zone (vertebral artery foramen), and lateral zone. The medial zone is believed to play an important role in the aetiology of cervical radiculopathy. Nobuhiro et al. [24] reported that the shape of the IVF approximates a funnel from lateral to medial with the entrance zone being the narrow part and the conically shaped radicular sheath with its take-off points from the central dural sac being the largest part. Consequently, nerve root compression occurs mostly in the narrow entrance zone of the IVF.

Extensive research has been conducted to investigate the IVF and nerve root in the lumbar spine. In the cervical region, however, most studies have focused on the evaluation of the vertebral body, spinal canal and spinal cord [26]. Nobuhiro et al. [24] stated that the cervical IVF is located under the isthmic region and it was divided into 3 zones: medial, middle, and lateral. When compared with the other 2 zones, the 
medial zone was believed to play an important role in the aetiology of cervical radiculopathy. In the present work the foramen is found to be bounded by the uncinate process anteriorly, the superior facet posteriorly and the adjacent pedicles superiorly and inferiorly.

The major factors contributing to a decrease in size of the IVF, as described in the medical literature on this particular issue, are degenerative reshaping of its bony borders, subluxation of the facet joints, decrease in height, protrusion of the intervertebral disc or bulging of the ligamentum flavum [6]. Information on the size of the osseous border of the IVF is limited. Furthermore, established clinical definitions for the assessment of IVF diameter cannot be applied easily on dry bone specimens [19]. The typical pear shape of the superior and inferior soft-tissue parts of the foramen in the living are different from their bony boundaries [4]. It is crucial to propose simple measurements especially of the osseous IVF dimensions. However in the present study no dry bone measurement has been done due to the lack of intervertebral disks which affect greatly the dimensions of the foramen.

Humphreys et al. [13] found that cervical IVF widths are larger in healthy individuals than in symptomatic persons using CT scans. It is found in the present work that the cervical IVF widths are larger in healthy individuals than in patients suffering from radiculopathy using both X-rays and CT scans. This supports the conclusion that reduction in the foramen width aggravates the state of nerve compression greatly.

In the present study the intervertebral foraminal areas of $\mathrm{C} 3-\mathrm{C} 4$ through $\mathrm{C} 6-\mathrm{C} 7$ are found to be very similar in normal (control) cases. The mean differences in the area between the right and left sides were less than $12 \%$ at all levels. The overall measurements in women were slightly smaller than in men (by $2 \%$ ). The greatest difference in height was $6.8 \%, 4.3 \%$ in width, and $7.1 \%$ in area. This agrees greatly with the work of Hogg-Johnson et al. [12] and Nabil et al. [22].

It is observed that the mean intervertebral foraminal area of C3-C4 of the right and left sides in females of the age group IV, is slightly increased in the pathological specimens (right: $0.73 \pm 0.04$, left: $0.77 \pm 0.04$ ), compared with the control group (right: $0.72 \pm 0.04$, left: $0.75 \pm 0.04$ ) with no statistical difference to be found. The same result was mentioned by Jiayong et al. [17]. This is suggestive of an increase in the intervertebral foraminal area in the cases suffering from neck pain, probably due to the increase in the size of one or more of the contents of the foramen. Moreover, this increase may be caused by degenerative changes in the bones due to the aging process and the pathological severity of the case. However, in males it is observed that the mean intervertebral foraminal area on both sides at all age groups, is less in pathological than in normal (control) group.

The present work proves a significant difference in the mean intervertebral foraminal area in the control group of $\mathrm{C} 5-\mathrm{C} 6$ and $\mathrm{C} 6-\mathrm{C} 7$ which was greater than that of $\mathrm{C} 3-\mathrm{C} 4$ and $\mathrm{C} 4-\mathrm{C} 5(\mathrm{p}<0.03)$. Although in the pathological cases no significant differences were found ( $p>0.05$ ). This could be explained by the presence of the roots of the brachial plexus in the lower foramina in normal group, while in the pathological cases it denotes narrowing of the lower foramina that causes the clinical symptoms and signs of radiculopathy. This result is supported in the work of Rubinstein et al. [28], Tong et al. [33] and Yue et al. [35], who reported that the main factor in the production of radiculopathies is narrowing of the intervertebral foramina. The results of the present study have also demonstrated statistically significant differences in the intervertebral foraminal area between right and left sides from C3-C4 all throw $\mathrm{C} 6-\mathrm{C} 7$, in both sexes, in control and pathological groups. This could be contributed to the size of the structures that are present in the IVF; many factors could play a role in this difference such as in the right handed persons, occupational and social differences of the cases [3].

Surgical procedures for cervical radiculopathy have been performed by either an anterior or posterior approach [16]. Although the anterior approach to the cervical spine has received the most attention in recent years. Exposure of the cervical nerve roots from the posterior approach provides the advantage of direct visualisation of the nerve root and the dura [14]. The posterior approach to the cervical spine for disc disease and spondylosis remains an important part of the spine surgeon's strategy [9]. However, anatomic studies on cervical foraminotomy are not well established and entail a deep understanding of the surgical anatomy of the intervertebral foramina [1]. Even in the conservative treatment of radiculopathy by traction the thoroughly knowledge of the IVF diameters in different age groups should be taken from the data obtained in this study which can be a useful guide.

\section{CONCLUSIONS AND RECOMMENDATION}

It is recommended to carry out a further study to clarify the relationship between the interverte- 
bral foraminal areas of the right or left handed individuals and investigate the sex differences of the studied measurements. Meanwhile, a complete personal history should be recorded (height, weight, occupation and lifestyle) of the individuals subjected to radiological examination of the cervical spine. This is to establish a database concerning the cervical intervertebral foraminal region size, variability, sex differences to determine the best line of treatment for each patient.

\section{REFERENCES}

1. Abbasi A, Malhotra G, Malanga G (2007) Complications of interlaminar cervical epidural steroid injections: a review of the literature. Spine, 32: 2144-2151.

2. Benjamin R, Singh V, Parr A (2009) Systematic review of the effectiveness of cervical epidurals in the management of chronic neck pain. Pain Physician, 12: 137-157.

3. Bryden H, Pamela J, Bruyn J, Fletcher PC (2005) Handedness and health: an examination of the association between different handedness classifications and health disorders. Laterality, 10: 429-440.

4. Carette S, Fehlings M (2005) Clinical practice. Cervical radiculopathy. N Engl J Med, 353: 392-399.

5. Charles DM, Lial ML, Schneider DI (1990) Fundamentals of College Algebra. $3^{\text {rd }}$ Ed. Scott Foresman, Little, p. 381.

6. Clark CR, Benzel EC, Currier BL (2005) The Cervical Spine Research Society Editorial Committee. The cervical spine. $4^{\text {th }}$ Ed. Lippincott Williams \& Wilkins, Philadelphia, PA, pp. 349-366.

7. Constantoyannis C, Konstantinou D, Kourtopoulos $\mathrm{H}$ (2002) Intermittent cervical traction for cervical radiculopathy caused by large-volume herniated disks. J Manipulative Physiol Therapy, 25: 188-192.

8. Czervionke LF, Daniels DL, Ho PS (1988) Cervical neural foramina: correlative anatomic and MR imaging study. Radiology, 169: 753-759.

9. Dreyfuss P, Baker R, Bogduk N (2006) Comparative effectiveness of cervical transforaminal injections with particulate and nonparticulate corticosteroid preparations for cervical radicular pain. Am Acad Pain Med, 7: 237-242.

10. Ebraheim NA, An HS, Xu R, Ahmad M, Yeasting RA (1996) The quantitative anatomy of the cervical nerve root groove and the intervertebral foramen. Spine, 21: 1619-1623.

11. Grundy PL, Germon TJ, Gill SS (2000) Transpedicular approaches to cervical uncovertebral osteophytes causing radiculopathy. J Neurosurg (Spine 1), 93: 21-27.

12. Hogg-Johnson S, Van der Velde G, Carroll $\sqcup$ (2008) The burden and determinants of neck pain in the general population: results of the bone and joint decade 2000-2010 task force on neck pain and its associated disorders. Spine, 33: S39-S51.

13. Humphreys SC, Chase J, Patwardhan A, Shuster J, Lomansey L, Hodges SD (1998) Flexion and traction effect on C5-C6 foraminal space. Arch Phys Med Rehabil, 79: 1105-1109.

14. Isu T (2002) Surgical selection for cervical spondylosis and cervical disc hernia. Jpn J Neurosurg, 11: 453-460.

15. Jahnke RW, Hart BL (1991) Cervical stenosis, spondylosis, and herniated disc disease. Radiol Clin North Am, 29: 777-791.
16. Jho HD, Kim MH, Kim WK (2002) Anterior cervical microforaminotomy for spondylotic cervical myelopathy: part 2. Neurosurgery, 51: S54.

17. Jiayong L, Nabil AE, Sanford CG (2008) Quantitative changes in the cervical neural foramen resulting from axial traction: in vivo imaging study. Spine, 8: 619-623.

18. Joghataei MT, Arab AM, Khaksar H (2004) The effect of cervical traction combined with conventional therapy on grip strength on patients with cervical radiculopathy. Clin Rehabil, 18: 879-887.

19. Kaplan M, Derby R (1998) Epidural corticosteroid injections: when, why, and how? J Musculoskel Med, 15: 39-46.

20. Lu J, Ebraheim NA, Huntoon M (2000) Cervical intervertebral disc space narrowing and size of intervertebral foramina. Clin Orthop Relat Res, 370: 259-264.

21. Moeti P, Marchetti G (2001) Clinical outcome from mechanical intermittent cervical traction for the treatment of cervical radiculopathy: a case series. J Orthop Sports Phys Ther, 31: 207-213.

22. Nabil AE, Jiayong L, Satheesh K (2009) Morphological changes in the cervical intervertebral foramen dimensions with unilateral facet joint dislocation injury. Int J Care Injured, 40: 1157-1160.

23. Narayan P, Haid RW (2001) Treatment of degenerative cervical disc disease. Neurol Clin, 19: 217-229.

24. Nobuhiro T, Yoshinori F, An H (2000) The anatomic relation among the nerve roots, intervertebral foramina, and intervertebral discs of the cervical spine. Spine, 25: 286-291.

25. Olivero WC, Dulebohn SC (2002) Results of halter cervical traction for the treatment of cervical radiculopathy: retrospective review of 81 patients. Neurosurg Focus, 12: Clinical part 1.

26. Panjabi MM (1998) Cervical spine models for biomechanical research. Spine, 23: 2684-2699.

27. Rao R (2003) Neck pain, cervical radiculopathy, and cervical myelopathy: pathophysiology, natural history, and clinical evaluation. Instr Course Lect, 52: 479-488.

28. Rubinstein S, Pool JJ, Tulder MW (2007) A systematic review of the diagnostic accuracy of provocative tests of the neck for diagnosing cervical radiculopathy. Eur Spine J, 16: 307-319.

29. Ruhli FJ, Muntener M, Henneberg M (2006) Human osseous intervertebral foramen width. Am J Phys Anthropol, 129: 177-188.

30. Ruggieri PM (1995) Cervical radiculopathy. Neuroimaging Clin N Am, 5: 349-366.

31. Selcuk YH, Kocaeli K, Aysun UZ (2003) Clinical importance of ligamentous and osseous structures in the cervical uncovertebral foraminal region. Clin Anat, 16: 404-410.

32. Sohn HM, You JW, Lee JY (2004) The relationship between disc degeneration and morphologic changes in the intervertebral foramen of the cervical spine: a cadaveric MRI and CT study. J. Korean Med Sci, 19: 101-106.

33. Tong H, Haig A, Yamakawa K (2002) The Spurling's test and cervical radiculopathy. Spine, 27: 156-159.

34. Wlodyka DS, Poiraudeau S, Catanzariti JF, Rannou F, Fermanian J, Revel M (2004) The ability to change of three questionnaires for neck pain. Spine, 71: 317-326.

35. Yue W, Tan SB, Tan MH (2001) The Torg-Pavlov ratio in cervical spondylotic myelopathy: A comparative study between patients with cervical spondylotic myelopathy and a nonsponylotic, nonmyelopathic population. Spine, 26: 1760-1764. 